\title{
EHMT2/G9a as an Epigenetic Target in Pediatric and Adult Brain Tumors
}

\author{
Barbara Kunzler Souza ${ }^{1,2, *}$, Natalia Hogetop Freire1, Mariane Jaeger ${ }^{1,3}$, Caroline Brunetto de Farias ${ }^{1,3}$, Algemir L. \\ Brunetto $^{1,3}$, André T. Brunetto ${ }^{1,3}$ and Rafael Roesler ${ }^{1,4, *}$ \\ 1. Cancer and Neurobiology Laboratory, Experimental Research Center, Clinical Hospital (CPE-HCPA) \\ Federal University of Rio Grande do Sul, 90035-003 Porto Alegre, RS, Brazil \\ Epigenica Biosciences, 92035-000 Canoas, RS, Brazil \\ Children's Cancer Institute, 90620-110 Porto Alegre, RS, Brazil \\ Department of Pharmacology, Institute for Basic Health Sciences, Federal University of Rio Grande do \\ Sul, 90050-170 Porto Alegre, RS, Brazil \\ * Correspondence: authors: barbara@epigenica.com.br (B.K.S.); rafaelroesler@hcpa.edu.br (R.R.).
}

\begin{abstract}
Epigenetic mechanisms, including post-translational modifications of DNA and histones that influence chromatin structure, regulate gene expression during normal development, and are also involved in carcinogenesis and cancer progression. The histone methyltransferase G9a (euchromatic histone lysine methyltransferase 2, EHMT2), which mostly mediates mono- and dimethylation by histone $\mathrm{H} 3$ lysine 9 (H3K9), influences gene expression involved in embryonic development and tissue differentiation. Overexpression of G9a has been observed in several cancer types, and different classes of G9a inhibitors have been developed as potential anticancer agents. Here, we review the emerging evidence suggesting the involvement of changes in G9a activity in brain tumors, namely glioblastoma (GBM), the main type of primary malignant brain cancer in adults, and medulloblastoma (MB), the most common type of malignant brain cancer in children. We also discuss the role of G9a in neuroblastoma (NB) and the drug development of G9a inhibitors. Finally, we present evidence that G9a inhibition dose-dependently reduces $\mathrm{MB}$ cell viability, and that transcriptional levels of $\mathrm{G} 9$ a are higher in $\mathrm{MB}$ tumors belonging to the $\mathrm{SHH}$, Group 3, and Group 4, compared to Wnt tumors. Importantly, our findings suggest that higher G9a gene expression may be a predictor of poor prognosis in patients with SHH MB.
\end{abstract}

Keywords: G9a; EHMT2; glioblastoma; medulloblastoma; epigenetics; brain tumor

\section{Introduction}

The concept of epigenetic regulation comprises heritable and non-heritable longterm changes in gene expression that are not dependent on mutations in DNA sequences. Epigenetic mechanisms that regulate gene expression, such as post-translational modifications of DNA and histones influencing chromatin structure, are now established players in cancer biology. Chromatin is organized in units called nucleosomes, each of which consist of a 146-bp segment of DNA wrapped around an octamer of histone proteins. Histone acetylation and deacetylation, and histone and DNA methylation act in concert to regulate the relaxation status of chromatin and transcriptional activity [1]. In cancer, chromatin condensation and DNA methylation can favor the repression of genes that promote differentiation and cell death, while promoting expression of genes related to cell survival, proliferation, and stemness. This reflects the view of cancer as a developmental disease, where carcinogenesis hijacks biological processes involved in normal embryonic development. Epigenetic programming orchestrates the different phases of tissue development, mediating, together with growth factors and other biochemical signals, the transition from 
a highly proliferative and non-differentiated state to later stages where differentiation and cell selection become key for the establishment of specialized cells and tissues from an original pluripotent cell. In cancer, the epigenetic machinery can be maintained in a state that keeps a less differentiated, stem-cell like, and highly proliferative phenotype. Thus, experimental epigenetic therapies are currently investigated with the aim of reverting the oncogenic epigenetic patterns of histone modifications and DNA methylation that maintain tumor malignancy. [2-4]. In brain tumors and other tumor types possibly originating from neuroectodermal or neural stem cells, abnormal epigenetic programming may stimulate cell survival and stemness, and epigenetic compounds can increase cell death and promote neural differentiation [5-10].

The best studied epigenetic modification in cancer is DNA methylation. It results in condensed chromatin structure and transcriptional inactivation, thus repressing or silencing genes. DNA methyltransferases (DNMTs) mediate DNA methylation that occurs mostly in DNA regions called "CpG islands", which concentrate CpG dinucleotides at gene promoters, regulatory regions, and gene bodies [11]. However, increasing attention has also been paid to histone methylation, which is mainly regulated by histone methyltransferases (HMTs). Methylation, as well as acetylation, phosphorylation, ubiquitination and ADP-ribosylation, modify the N-terminal tails of core histones, resulting in relaxation or condensation of chromatin due to changes in the affinity between histones and DNA, or regulating gene expression by interfering with the binding of transcription factors to DNA sequences $[12,13]$. Histone methylation is a reversible post-translational modification where up to three methyl groups can be added to a histone lysine residue and up to two groups to a histone arginine residue, resulting in an epigenetic mark that can either activate or repress overall levels of gene expression [14].

Methylation of $\mathrm{H} 3$ lysine 9 (H3K9) is a hallmark of heterochromatin, the condensed, transcriptionally inactive state of chromatin. Mono- and dimethylation of H3K9 are mediated by the HMT G9a (euchromatic histone lysine methyltransferase 2, EHMT2) [15]. G9a is encoded by the Ehmt2 gene, located in the major histocompatibility complex (MHC) locus in mice and human leukocyte antigen (HLA) locus in humans, and contains 28 exons that code for a 1,263 amino acid nuclear protein belonging to the Su(var)3-9 family $[16,17]$. The different domains comprising G9a are a catalytic SET domain, a domain containing ankyrin repeats involved in protein-protein interactions, and nuclear localization signals on the N-terminal region (Figure 1). However, G9a does not contain a DNAbinding domain, requiring cofactors for its localization to specific genes [18-24]. Through its methyltransferase activity, as well as methyltransferase-independent actions mediated by its N-terminal domain [23, 25, 26], G9a regulates changes in gene expression involved in embryonic development and differentiation of normal tissues [19-21, 24].

Overexpression of G9a is found across different solid tumor types including lung, ovarian, esophageal, hepatocellular, and brain cancers, as well as in multiple myeloma, and has been associated with poor prognosis in several cancer types [27-29]. Higher G9a levels are associated with increased methylation that inhibits the expression of tumor suppressor genes, likely resulting in more aggressive phenotypes, with increased invasiveness and metastasis [30-32]. For example, small interfering RNA (siRNA)-mediated G9a knockdown rescues the expression of the tumor-suppressor gene MASPIN in MDA-MB231 breast cancer cells [31]. Expression of G9a is higher in metastatic lesions compared to their corresponding primary tumors in ovarian cancer, and knockdown of G9a inhibits prometastatic cellular activities while G9a over-expression promotes these cellular properties [30]. In this review, we will focus on the role of G9a as an epigenetic regulator and pro-tumoral controller in brain tumors. 


\section{Figure 1}

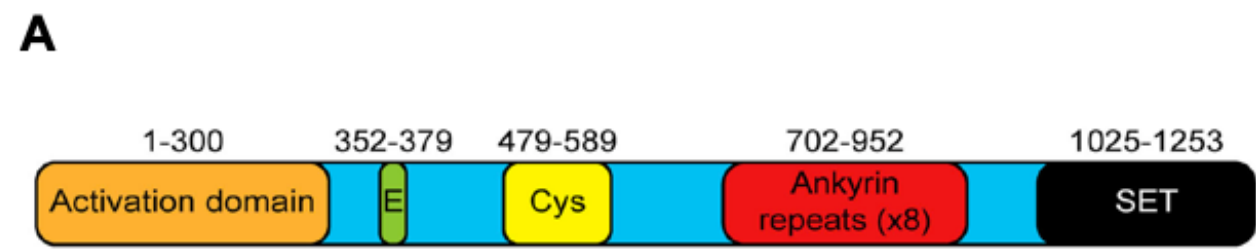

Figure 1. Structure of G9a. The protein contains 1,253 amino acids and distinct domains including an N-terminal activation domain, glutamate-rich (23 consecutive Glu residues) and cysteine-rich regions, eight ankyrin repeat units (binding of dimethylated lysine residues), and a C-terminal enzymatic SET domain [23].

\section{G9a and Glioma}

Glioblastoma (GBM) is the most aggressive type of primary malignant brain tumor in adults, and also presents pediatric types, such as diffuse intrinsic pontine glioma and pediatric non-brainstem high-grade glioma. GBM can be a primary tumor or develop as secondary GBM from lower-grade tumors harboring a mutation in isocitrate dehydrogenase (IDH). Current treatment based on combining surgical resection followed by radiotherapy and chemotherapy results in a median overall survival of less than 2 years, and the development of novel, molecularly-targeted therapies, is urgently needed [33-36]. It has been less than 10 years since the first evidence of a role for G9a in GBM and gliomas of lower grades started to emerge. H3K9me3, a marker of G9a-mediated repression of transcription, is found across different types of astrocytic tumors ( $48 \%$ of pilocytic astrocytomas, $78 \%$ of diffuse astrocytomas, $67 \%$ of anaplastic astrocytomas, and $79 \%$ of GBMs) and is significantly associated with IDH mutations in oligodendrogliomas. Combined H3K9me3 positivity and $1 \mathrm{p} 19 \mathrm{q}$ codeletion is found in most World Health Organization (WHO) grade II and grade III oligodendrogliomas. H3K9me3 is a prognostic marker of improved overall survival in grade II oligodendrogliomas compared with $\mathrm{H} 3 \mathrm{~K} 9$ me3-negative cases. These findings suggest that H3K9me3 may define a subset of tumors with better overall survival in grade II oligodendrogliomas, but not in higher grade gliomas [37]. Thus, G9a may have a complex role where, at least in some selected cancer types, its expression is related to a better rather than worst prognosis.

Changes in expression of genes coding for lysine and arginine methyltransferases including G9a play a role in GBM pathogenesis [38]. An immunohistochemical study aimed at investigating G9a, H3K9me2 and histone H3K9me1 in human glioma and adjacent non-neoplastic tissue samples found that $86 \%$ (43 of 50) are positive for G9a expression, compared to $42 \%$ (21 of 50 samples) in non-neoplastic tissues. Expression of G9a and H3K9me2 are positively associated with a higher WHO glioma grade. and the G9a inhibitor BIX-01294 inhibits proliferation, induces apoptosis, and reduces mono- and dimethylation of H3K9 in U251 human GBM cells [39].

A network of epigenetic components including G9a regulates gene expression in GBM cells. G9a can form a protein complex with a DNMT to epigenetically regulate the $N Y$-ESO1 gene, which is a is a cancer/testis antigen proposed as a suitable target for cancer immunotherapy [40]. G9a is highly expressed in GBM cells, and pharmacological inhibition or genetic knockdown of G9a reduces cell proliferation and tumorigenesis in experimental GBM in vitro and in vivo. Knockdown of G9a also results in cell cycle arrest in the G2 phase accompanied by reduced expression of CDK1, CDK2, Cyclin A2, and Cyclin B1 
and activation of autophagy [41]. The G9a inhibitor BIX01294 triggers apoptosis and autophagy, increases apoptosis markers cleaved caspase 3, caspase 7 and poly (ADP-ribose) polymerase (PARP), and reduces H3K9 dimethylation (H3K9me2) in cultured GBM cells, in addition to promoting differentiation of GBM stem cells [42]. The same inhibitor also sensitizes GBM cells to the effects of the cytotoxic chemotherapeutic temozolomide, in terms of reduced cell viability, apoptotic-like alterations in morphology, and increase in apoptosis markers $[43,44]$, in addition to radiosensitizing GBM cells and inhibiting DNA double-strand breaks through both the homologous recombination and nonhomologous end-joining pathways [45].

Both pharmacological inhibition and siRNA-mediated knockdown of G9a increases the content of the autophagy marker LC3B in GBM cells [46]. G9a and the related methyltransferase protein, GLP directly bind to the $\alpha$ subunit of HIF-1 (HIF- $1 \alpha$ ) and catalyze mono- and di-methylation of HIF- $1 \alpha$ at lysine (K) 674 in vitro and in vivo [47]. The effects of G9a are at least partially mediated by the long non-coding RNA HOTAIRM1, which transcribes from the antisense strand of HOXA gene cluster which locus in chromosome 7p15.2. HOTAIRM1 functions as oncogenic factor regulating HOXA1 gene expression and sequestering G9a and DNMTs away from the HOXA1 gene promoter [48].

In tumors, epigenetic programming is crucial for maintenance of a stem-cell phenotype, with high proliferation but inhibited differentiation. Interferon $\gamma$ (IFN $\gamma$ ) increases H3K9me2 and reduces G9a levels, whereas G9a inhibition increases IFN $\gamma$ while reducing retinoic acid inducible gene (RIG-I) in GBM cells. These findings indicate a concerted interplay between G9a and the PPAR gamma coactivator- 1 alpha (PGC- $1 \alpha$ ) to promote RIGI-induced maintenance of a stem cell-like state in GBM cells [49]. One study [50] found that most GBM cells positive for expression of the stem cell marker CD133 are negative for G9a-dependent H3K9me2. Pharmacological inhibition of G9a stimulates sphere formation (an index of cancer stem cell expansion in vitro) and increases expression of stem cell markers CD133 and Sox2 in GBM cell cultures. Conversely, overexpression of G9a increases $\mathrm{H} 3 \mathrm{~K} 9 \mathrm{me} 2$ and reduces sphere formation along with CD133 and Sox2 content. Moreover, the authors confirmed that H3K9me2 acts on CD133 and Sox2 promoter regions to repress their expression. Together, these findings strongly indicate that, although G9a may function as a promoter of brain tumor progression through maintenance of a stem cell-like phenotype, at least under some conditions it can also inhibit brain tumor stem cell formation. Expression of genes related to autophagy and differentiation is upregulated by G9a inhibition via BIX01294 in putative glioma stem cells from spheres [42]. BIX01294 enhances the cytotoxic effects of temozolomide in GBM stem-like cells, even in the absence of changes in pluripotency markers NANOG, SOX2, and CD133, or methylation of NANOG and SOX2 gene promoters. These results suggest a potential role for G9a inhibition as a component of a combination therapy strategy to increase the efficacy and reduce resistance to cytotoxic chemotherapeutics [43].

\section{G9a and Medulloblastoma}

In children, brain tumors are the most common solid tumors and the leading cause of cancer-related death. Medulloblastoma (MB) is the most common type of malignant brain cancer of the childhood and can more rarely occur in adults as well. Remarkable advancements in our understanding of MB biology have been made during the past decade. The current molecular classification divides MB tumors into four consensus subgroups with distinct genomic, epigenetic, and clinical features: WNT, SHH, Group 3, and Group 4 [51,52]. This molecular classification has rapidly made its translation to the clinical setting, becoming important for guiding patient risk stratification, treatment, and selection in clinical trials $[53,54]$. Two subgroups are defined by mutations leading to aberrant activation of the Wingless (WNT) and Sonic hedgehog (SHH) pathways, whereas Group $3 \mathrm{MB}$ display amplification of genes involved in the Notch and transforming growth factor- $\beta$ (TGF $\beta$ ) pathways, and Group 4 show a prominent representation of genes 
involved in regulating chromatin state [51, 55]. Group 3 and Group 4 MBs are more aggressive and associated with a poor prognosis, with patients with Group $3 \mathrm{MB}$ often showing metastasis at diagnosis and having a 5-year survival of around $50 \%[53,55,56]$. Recent descriptions of intra- and intertumoral cellular and molecular heterogeneity within subgroups have led to further classifying MB into 12 subtypes. Although multimodal treatment with surgery radiotherapy, and chemotherapy has improved cure rates, about onethird of patients relapse, and survivors experience long-lasting neurological, cognitive, and endocrinological sequelae. Thus, the development of molecularly targeted therapies is urgently needed [55-58].

The deubiquitylase USP37 regulates cell proliferation by acting on the stability of the cyclin-dependent kinase inhibitor 1B (CDKN1B/p27Kip1)., and may act as a tumor suppressor in MB, Expression of USP37 is downregulated in human MB, and USP37 reduces the growth of experimental MB. G9a reduces USP37 expression by promoting H3K9 mono-, di-, and trimethylation at its promoter. The USP37 promoter show a considerable level of H3K9 trimethylation, which is reduced after inhibition of G9a activity. These findings reveal a role for G9a as an epigenetic regulator of G9a MB. In addition, the G9a inhibitor UNC0638 impairs proliferation of human DAOY MB cells and MB growth in vivo through mechanisms that include downregulating USP37 [59, 60].

G9a shows interplays with another histone methyltransferase, EZH2, often constituting an "axis" of functionally related epigenetic modifiers influencing cancer cells. EZH2 has been shown to play a role in GBM and MB, and EZH2 inhibitors have been evaluated in preclinical models of brain cancers. For example, the pyrazole compound MC3629, developed as an analog of two different SAM-competitive EZH2 inhibitors, EPZ005687 and GSK2816126, has been shown as an inhibitor of G91/EZH2 signaling in MB capable of impairing cell proliferation, stemness, and $\mathrm{H} 3 \mathrm{~K} 27 \mathrm{me} 3$, and inducing apoptosis in human MB cell models representing the SHH group, as well as in a mouse model of SHH MB xenografts. Moreover, MC3629 showed good blood-brain barrier penetration and was able to reduce H3K27me3 levels in the brain and cerebellum in mice [61].

$\mathrm{MB}$ cell lines representative of subgroups $\mathrm{SHH}$ and 3/4 show detectable levels of both G9a and its repressive mark H3K9me2, and G9a inhibition by UNC0638 dose-dependently reduces MB cell viability (Figure 2). Also, we have performed analyses of G9a gene expression in a set of 763 patients with $\mathrm{MB}$ tumors belonging to different molecular subgroups (described by Cavalli and collaborators, [62]). The findings reveal that G9a levels are higher in tumors belonging to the SHH group, Group 3, and Group 4, compared to Wnt tumors, which show better prognosis compared to all other groups (Figure 3A). Remarkably, G9a expression may predict prognosis in MB patients, with higher expression indicating shorter overall survival across the four molecular subgroups, although a statistically significant difference is specifically observed in subgroup SHH (Figure 3B). These findings reveal for the first time the exciting possibility that analyzing the expression of the G9a gene may provide a novel prognostic marker for overall survival in patients with SHH MB. 


\section{Figure 2}
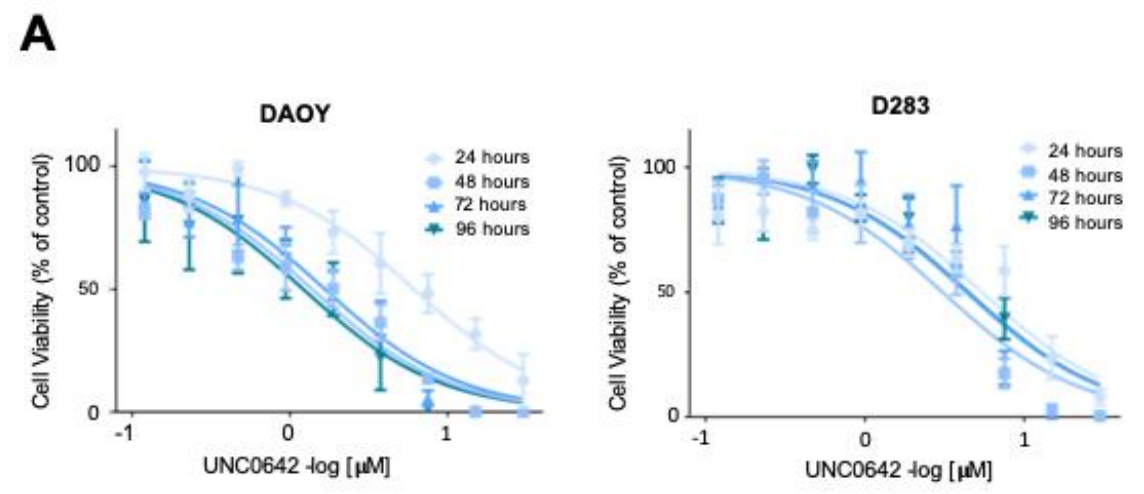

B

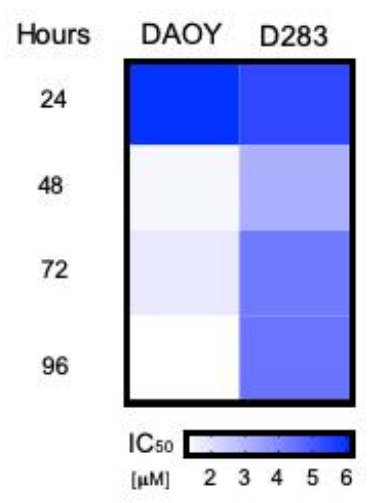

C

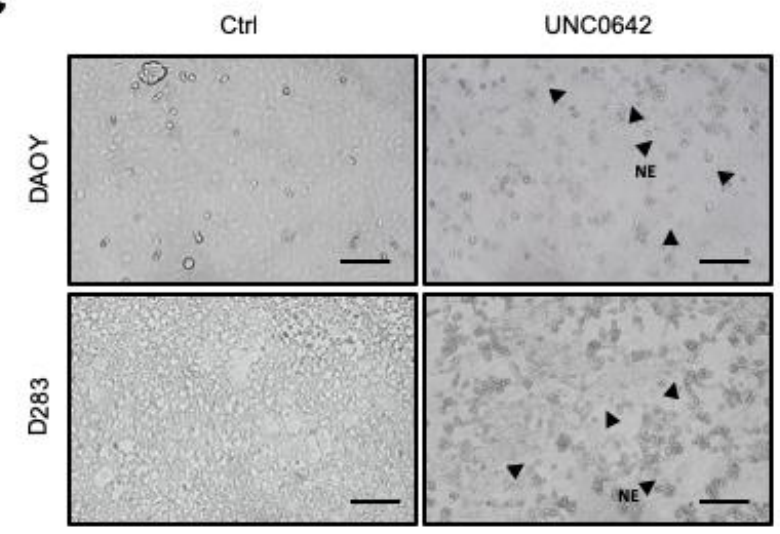

Figure 2. G9a inhibition impairs the viability of human MB cells. A. Cell viability of MB cell lines DAOY and D283 treated using 9 concentrations $(0.1-30 \mu \mathrm{M})$ of UNC0642 for 24, 48, 72 and 96 hours. Cell viability was assessed using the trypan blue exclusion method in the Neubauer chamber as previously described [95]. All assays were performed in triplicate and repeated in three independent sets. For calculation of IC 50 , data were fitted in a dose response curve (Graphpad Prism v. 6.0) using the equation: $y=\min +(\max -\min ) /\left(1+10^{\wedge}\left((\log I C 50-x)^{*}\right.\right.$ Hillslope $\left.\left.+\log ((\max -\min ) /(50-\min )-1)\right)\right)$. B. Heatmap showing $\mathrm{IC}_{50}(\mu \mathrm{M})$. The color key represents the average $\mathrm{IC}_{50}(\mu \mathrm{M})$ values: White color indicates low IC $\mathrm{C}_{50}$ whereas blue color represents high IC 50 . The average IC 50 was calculated based on the cell count by Trypan exclusion method of 3 biological replicates across $2 \mathrm{MB}$ cell lines at 24, 48, 72 and 96 hours using GraphPad Prism. C. An illustrative image showing that UNC0642 in vitro reduced cell growth in DAOY and D283 MB cell lines after treatment with $3.8 \mu \mathrm{M}$ and $7.5 \mu \mathrm{M}$ at 96 hours, respectively. Scale bars, $200 \mu \mathrm{m}$. 


\section{Figure 3}

A

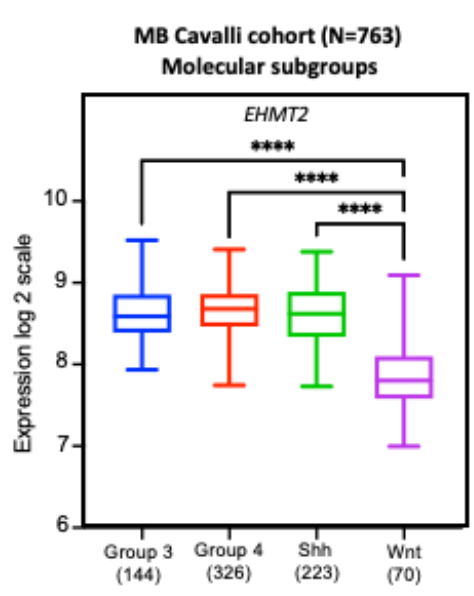

B

MB Cavalli cohort
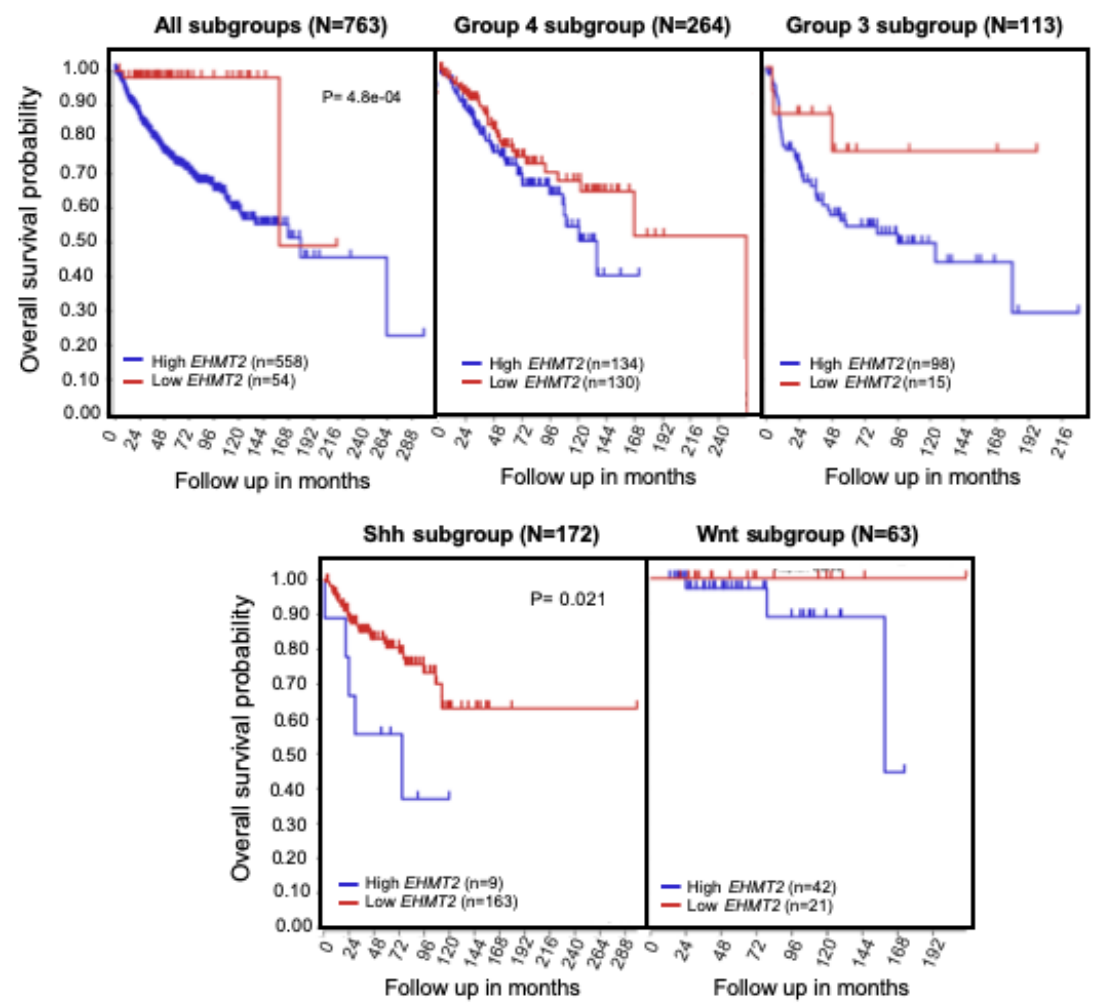

Figure 3. Transcript levels of G9a in tumors from patients with different molecular subgroups of MB. G9a expression levels were examined in previously described transcriptome data sets comprising a total of 763 medulloblastoma patient samples, from Cavalli cohort ( $\mathrm{n}=763$ samples profiled on the Affymetrix Gene $1.1 \mathrm{ST}$ array as previously described and normalized using the RMA method) [62]. Expression of the markers across all samples was normalized within the R2: Genomics Analysis and Visualization Platform (http://r2.amc.nl) and presented in box plot format as log2-transformed signal 
intensity. All subgroups and subtypes of each data set were compared using a Kruskal-Wallis test for significance Tukey's test for post-hoc analysis, using GraphPad Prism. $\mathrm{p} \leq 0.001$ was considered statistically significant. Overall survival (OS) was measured from the time of initial diagnosis to the date of death or the date of last follow up, using combined OS and gene expression data from all datasets selected. Survival distribution was estimated according to the Kaplan-Meier method using a median cut-o_ and log-rank statistics; $P \leq 0.05$ was considered statistically significant.

\section{G9a and Neuroblastoma}

Neuroblastoma (NB) arises commonly in the peripheral nervous system, from embryonal neural crest cells that later give rise to the sympathetic nervous system, and is thus not commonly classified as a brain tumor $[63,64]$. However, it can also rarely occur intracranially [65-67], and will thus be included in the present review. NB accounts for around $15 \%$ of cancer-related deaths in children and adolescents. High-risk NB, usually defined by amplification of the MYCN oncogene, confers a poor prognosis with short overall survival [64]. NB shows an abnormal epigenetic reprogramming, with DNA methylation-induced repression of tumor suppressing genes [68, 69].

A microarray-based search of tumors from 88 patients showed that low G9a expression is correlated with better overall survival, whereas, in contrast, high G9a expression is associated with a poor outcome. Expression of G9a varies with tumor stage, where expression is higher in stage 4 tumors compared to stages 3 and 4S. Furthermore, G9a expression is significantly higher in patients having the NB tumor as cause of death compared to the non-death group. Treatment of NB cell lines were treated with the G9a inhibitor BIX01294 resulted in a reduction in both cell proliferation in vitro and tumorigenicity in NOD/SCID mice, in addition to the appearance of markers of autophagy. Finally, similar findings are observed in cells submitted to G9a knockdown with lentiviral human G9a shRNA [70]. Treating human SH-SY5Y NB cells with the estrogenic endocrine disruptor bisphenol A led to a reduction in $\mathrm{H} 3 \mathrm{~K} 9 \mathrm{me} 3$ and changes in expression of G9a among other chromatin modifiying genes [71].

Immunological analysis showed G9a protein to be more highly expressed in NB cell lines harboring $M Y C N$ amplification, a crucial marker of poor outcome in NB patients. In primary NB tumors, the content of G9a protein is higher in poorly differentiated or undifferentiated tumors and correlates with expression of the related oncoprotein EZH2. G9a depletion via use of siRNA, or treatment with G9a inhibitors, UNC0638 and UNC0642, reactivates tumor suppressor genes, reduces NB cell proliferation and induces apoptosis, but these effects are observed selectively in cells with $M Y C N$ amplification. The need for MYCN for G9a inhibition to be effective is also demonstrated in the SHEP-21N isogenic model with tet-regulatable MYCN [72]. G9a and GLP are repressors of transcriptional responses to interferon- $\gamma$ (IFN- $\gamma$ ) in NB cells. Inhibition of G9a and GLP enhances IFN- $\gamma-$ induced expression of the Th1-type chemokines CXCL9 and CXCL10, which crucially mediate recruitment of T-cells to the tumor microenvironment. G9a inhibition is required for transcriptional responses to IFN- $\gamma$ and histone mark changes at CXCL9 and CXCL10 gene loci in $M Y C N$-positive NB cells. These findings highlight the important role of G9a in high-risk, MYCN-expressing, NB [73].

\section{Drug Development of G9a Inhibitors for Cancer Therapy}

The small molecule compound BIX01294, which displays inhibitory activity on both G9a and GLP, is an early inhibitor developed about a decade ago that represents a 2,4diamino-6,7-dimethoxyquinazoline template. Building upon the same template, compound 10 (UNC0224) was developed as another G9a inhibitor with excellent selectivity and potency. Structural insight from the X-ray crystal structure of the G9a-10 complex enabled optimization of the 7-dimethylaminopropoxy side chain of UNC0224 resulted in the discovery of compound 29 (UNC0321), the first G9a inhibitor with potency at picomo- 
lar concentrations [74]. Further developments by the same group, for the design new generations of analogues aimed at improving cell membrane permeability, led to the development of UNC0646 and UNC0631, which show excellent potency in cells as well as excellent relationship between potency and cytotoxicity [75]. More recently, the 2-alkyl-5amino- and 2-aryl-5-amino-substituted $3 \mathrm{H}$-benzo[e][1,4]diazepine scaffold was used to identify compound 12a (EML741), which displays high in vitro and cellular potency comparable to BIX01294 inhibitory activity against DNMT1 [76]. Replacing the hydrophobic segment of BIX-01294 and UNC0638 with a guanidine moiety (side-chain moiety of arginine) resulted in additional compounds. The guanidine moieties were positioned similar to the Arg8 of the substrate peptide in molecular docking, and reactivity of the guanidinesubstituted inhibitors was observed in density functional theory studies. Molecular dynamics, molecular mechanics Poisson-Boltzmann surface area binding free energy, linear interaction energy, and potential mean force calculated from steered molecular dynamics simulations showed improved conformational stability and improved H-bond potential and binding affinity of the new compounds. In conclusion, the authors propose incorporating a guanidine group to mimic the substrate arginine side chain and improve the potency of G9a inhibitors [77].

An alkaloid derived from Chaetomium minutum, chaetocin, which is a structurally complex epidithiodiketopiperazine (ETP) showed to be a potent G9a inhibitor. Synthetic approaches aimed at discovering other G9a inhibitors based on structure-activity relationships of chaetocin derivatives resulted in novel derivative PS-ETP-1 [78]. Another alkaloid, protoberberine alkaloid pseudodehydrocorydaline (CT13) was identified as a novel G9a inhibitor by structure-based virtual screening of an in-house library containing natural products. The activity of CT13 was determined by mass spectrometry and Western Blot analysis, and the compound displays selective inhibition of G9a in human breast cancer cells. Molecular docking indicated that CT13 acts on the histone H3 binding site [79].

Six novel analogs were designed with 3D quantitative structure-activity relationship (3D-QSAR) analysis of a series of 2,4-diamino-7-aminoalkoxyquinazolineas G9a inhibitors. Structural requirements for substituted 2,4-diamino-7-aminoalkoxyquinazoline for G9a inhibitory activity could be obtained from comparative molecular similarity indices analysis (COMSIA) plots [80]. Through structure-based virtual screening, DCG066 was developed as a new G9a inhibitor with a molecular scaffold distinct from previous inhibitors. This compound binds G9a directly and inhibits its activity in vitro in addition to reducing H3 methylation [81]. CPUY074020, based on the structure of UNC0638 and containing a $6 \mathrm{H}$-anthra[1,9-cd]isoxazol-6-one scaffold, was developed as a leading compound displaying potent dual G9a inhibitory and anti-proliferative activities, and also promoted cell apoptosis and reduced dimethylation of H3K9. Moreover, this compound shows good pharmacokinetic properties in vivo [82]. Structure-based design, synthesis, and screening of small molecules for dual inhibitory activity of G9a and histone deacetylases (HDACs) allowed the discovery of compound 14, which inhibits both enzymes in the low micromolar range in cell-based platforms [83]. Structure-based approaches also led to the design and synthesis of reversible chemical probes that inhibit both G9a and DNMTs at nanomolar ranges, resulting in compounds with in vitro antiproliferative activities in the nanomolar range and tumor growth inhibition in a human acute myeloid leukemia mouse model [84].

Molecular dynamics simulation and free energy calculations of five different modified/mutated G9a substrate peptides, in addition to evaluation of the binding energy contribution-based architecture of the active site of G9a was used to detail the molecular basis of G9a binding to inhibitors. These experiments revealed, for example, that Arg8 of the substrate peptide is crucial for determining binding to G9a. The G9a active site is segregated into energy rich and low regions, and the energy rich region is used for binding inhibitors. The findings suggested that potential G9a inhibitors would preferentially interact with residues Asp1074, Asp1083, Leu1086, Asp1088, Tyr1154 and Phe1158 og G9a 
[85]. Further structure-based analysis of activity cliffs, scaffold hops and G9a inhibitors with docking followed by molecular dynamics simulations confirmed the identification of these key residues [86].

Recent advances include the discovery, through a virtual screening strategy, of CSV0C018875, a novel quinoline-based G9a inhibitor. Also, sub-structure querying based on previous G9a inhibitors together with docking-based virtual screening, led to the identification of CSV0C018875, which, according to molecular dynamics simulations, binds deeper inside the active site of G9a, likely enabling tighter binding and a longer time of action [87].

\section{Conclusions and Future Directions}

The treatment of brain tumors remains one of the most challenging unmet medical needs of our time. In the case of GBM, for example, the virtual absence of pharmacological options and the lack of curative treatment protocols, combined with the very poor prognosis with a short life expectancy after diagnosis make absolutely urgent to search for novel therapeutics. With $\mathrm{MB}$, more effective and less toxic treatments are needed to improve the outcome of pediatric and adult patients with this tumor that represents one of the most important causes of disease-related death in children.

Given the extensive epigenetic reprogramming present in brain tumors, which redefines gene expression in an aberrant way to enhance cell proliferation, survival, and stemness while inhibiting apoptosis and differentiation, to help establish the malignant phenotype seen in these cancers, molecular components of epigenetic regulation have increased being highlighted as targets. In comparison to genetic mutations and other changes in DNA sequence, the epigenome provides more druggable targets, given the difficulty of directly manipulating DNA with pharmacological agents. And, compared to more specific molecular targets related to cell signaling, such as growth factor receptors and protein kinases, manipulating epigenetic processes may result in broader changes in gene expression, altering transcription of several sets of genes, possibly leading to a larger modification of the cellular phenotype. Moreover, given the increasingly recognized intertumoral and intratumoral complexity in terms of genetic and biochemical heterogeneity [62], epigenetic drugs could hypothetically be able to produce effects in cancer cells and tumors that show differences in genetic mutations or activation of signaling pathways. Based on the previous experience with epigenetic modulators in experimental cancer, it is possible that, in various circumstances, combination therapies using one G9a inhibitor plus cytotoxic chemotherapy, other epigenetic agents, or other classes of targeted agents, will be revealed as more effective than single-agent therapies focusing on G9a inhibition alone.

As seen above, increasing, although relatively sparse evidence suggests a role for G9a as a potential epigenetic molecular target in at least some types of adult and pediatric brain cancers, namely, GBM and $\mathrm{MB}$, in addition to $\mathrm{NB}$, a neural tumor that can rarely occur intracranially. To our knowledge, to date there is no evidence of a role for G9a in other brain tumor types, such as ependymoma and meningioma. Importantly, we report data indicating that G9a transcription levels may be a prognostic marker of lower overall survival, at least in patients with SHH tumors. Although this early finding needs to be confirmed by further analyses using larger tumor samples, it raises the possibility that G9a expression levels could be used by neuro-oncologists to predict whether patients with SHH MB tumors are likely to show a poorer or better prognosis in terms of overall survival.

We also present data showing that selective pharmacological inhibition of G9a consistently and dose-dependently impairs viability of DAOY and D283 human MB cells. D283 cells display MYC amplification and show features of groups 3 and $4 \mathrm{MB}$, whereas DAOY cells are TP53-mutated and represent SHH MB $[6,88]$. As seen above, several G9a inhibitors have been put forward as potential anticancer agents, and research efforts have 
been successful in developing effective small molecule agents. Compounds tested in experimental brain tumor models include molecules based on different chemical scaffolds including diazepinquinazolin-amines, benzimidazoles, natural products of chitin, and others. [89-91]. UNC0638 and other inhibitors are examples of a class of substrate competitive inhibitors, which directly occupy the binding site of the histone to G9a and specifically bind the substrate site in G9a, whereas other compounds constitute a second class of agents that act as competitive inhibitors of the S-adenosyl-methionine (SAM) cofactor [89]. Molecular docking experiments have identified compounds including ninhydrin, naphthoquinone, cysteamine and disulfide cysteamine as candidate G9a and GLP inhibitors [92]. In spite of these considerable advances in drug discovery, to date G9a inhibitors have not been evaluated in clinical trials [93, 94]. Further research should increase our understanding by focusing on identifying the possible differential sensitivities of distinct brain tumor molecular subgroups and subtypes to G9a inhibition, as well as comparing different available inhibitors, including dual inhibitors, in brain tumor models, in addition to evaluating combinations of G9a inhibitors with other epigenetic or non-epigenetic anticancer agents.

Author Contributions: B.K.S. and N.H.F. carried out the experiments and gene analyses shown in Figures 2 and 3. All authors contributed to the conception, writing, and revision of this article.

Funding and Support: The writing of this article was supported by the National Council for Scientific and Technological Development (CNPq, MCTI, Brazil) grants 407765/2017-4 and 305647/20199 and (R.R.); Rio Grande do Sul State Research Foundation (FAPERGS, RS, Brazil) grant 17/25510001 071-0 (R.R.); the Children's Cancer Institute; Epigenica Biosciences (B.K.S.).

Conflicts of Interest: B.K.S. is co-founder and CEO of Epigenica Biosciences Ltd. The authors declare no other conflicts of interest.

\section{References}

1. Kouzarides, T. Chromatin modifications and their function. Cell 2007, 128, 693-705. doi: 10.1016/j.cell.2007.02.005

2. Bates, S.E. Epigenetic therapies for cancer. N. Engl. J. Med. 2020, 383, 650-663. doi: 10.1056/NEJMra1805035

3. Baxter, E.; Windloch, K.; Gannon, F.; Lee, J.S. Epigenetic regulation in cancer progression. Cell Biosci. 2014, 4, 45. doi:10.1186/2045-3701-4-45

4. Ellis, L.; Atadja, P.W.; Johnstone, R.W. Epigenetics in cancer: targeting chromatin modifications. Mol Cancer Ther. 2009, 8, 14091420. doi: 10.1158/1535-7163.MCT-08-0860

5. Abballe, L.; Miele, E. Epigenetic modulators for brain cancer stem cells: Implications for anticancer treatment. World J. Stem Cells 2021, 13, 670-684. doi: 10.4252/wjsc.v13.i7.670

6. Jaeger, M.C.; Ghisleni, E.C.; Cardoso, P.S.; Siniglaglia, M.; Falcon, T.; Brunetto, A.T.; Brunetto, A.L.; de Farias, C.B.; Taylor, M.D.; Nör, C.; et al. HDAC and MAPK/ERK inhibitors cooperate to reduce viability and stemness in medulloblastoma. J. Mol. Neurosci. 2020, 70, 981-992. doi: 10.1007/s12031-020-01505-y

7. Lawlor, E.R.; Thiele, C.J. Epigenetic changes in pediatric solid tumors: promising new targets. Clin. Cancer Res. 2012, 18, 27682779. doi: 10.1158/1078-0432.CCR-11-1921

8. Nör, C.; Sassi, F.A.; de Farias, C.B.; Schwartsmann, G.; Abujamra, A.L.; Lenz, G.; Brunetto, A.L.; Roesler, R. The histone deacetylase inhibitor sodium butyrate promotes cell death and differentiation and reduces neurosphere formation in human medulloblastoma cells. Mol. Neurobiol. 2013, 48, 533-543. doi: 10.1007/s12035-013-8441-7

9. Perla, A.; Fratini, L.; Cardoso, P.S.; Nör, C.; Brunetto, A.T.; Brunetto, A.L.; de Farias, C.B.; Jaeger, M.; Roesler, R. Histone deacetylase inhibitors in pediatric brain cancers: biological activities and therapeutic potential. Front. Cell Dev. Biol. 2020, 8, 546. doi: $10.3389 /$ fcell.2020.00546

10. Souza, B.K.; da Costa Lopez, P.L.; Menegotto, P.R.; Vieira, I.A.; Kersting, N.; Abujamra, A.L.; Brunetto, A.T.; Brunetto, A.L.; Gregianin, L.; de Farias, C.B.; et al. Targeting histone deacetylase activity to arrest cell growth and promote neural differentiation in Ewing sarcoma. Mol. Neurobiol. 2018, 55, 7242-7258. doi: 10.1007/s12035-018-0874-6

11. Lujambio, A.; Portela, A.; Liz, J.; Melo, S.A.; Rossi, S.; Spizzo, R.; Croce, C.M.; Calin, G.A.; Esteller, M. CpG island hypermethylation-associated silencing of non-coding RNAs transcribed from ultraconserved regions in human cancer. Oncogene 2010, 29, 6390-6401. doi: 10.1038/onc.2010.361

12. Morera, L.; Lübbert, M.; Jung, M. Targeting histone methyltransferases and demethylases in clinical trials for cancer therapy. Clin. Epigenetics 2016, 8, 57. doi: 10.1186/s13148-016-0223-4

13. Yi, X.; Jiang, X.J.; Li, X.Y.; Jiang, D.S. Histone methyltransferases: novel targets for tumor and developmental defects. Am. J. Transl. Res. 2015, 7, 2159-2175. 
14. Lehnertz, B.; Ueda, Y.; Derijck, A.A.; Braunschweig, U.; Perez-Burgos, L.; Kubicek, S.; Chen, T.; Li, E.; Jenuwein, T.; Peters, A.H. Suv39h-mediated histone H3 lysine 9 methylation directs DNA methylation to major satellite repeats at pericentric heterochromatin. Curr. Biol. 2003, 13, 1192-1200. doi: 10.1016/s0960-9822(03)00432-9

15. Tachibana, M.; Ueda, J.; Fukuda, M.; Takeda, N.; Ohta, T.; Iwanari, H.; Sakihama, T.; Kodama, T.; Hamakubo, T.; Shinkai, Y. Histone methyltransferases G9a and GLP form heteromeric complexes and are both crucial for methylation of euchromatin at H3-K9. Genes Dev. 2005, 19, 815-826. doi: 10.1101/gad.1284005

16. Brown, S.E.; Campbell, R.D.; Sanderson, C.M. Novel NG36/G9a gene products encoded within the human and mouse MHC class III regions. Mamm. Genome 2001, 12, 916-924. doi:10.1007/s00335-001-3029-3

17. Milner, C.M.; Campbell, R.D. The G9a gene in the human major histocompatibility complex encodes a novel protein containing ankyrin-like repeats. Biochem. J. 1993, 290(Pt 3), 811-818. doi:10.1042/bj2900811

18. Bittencourt, D.; Wu, D.Y.; Jeong, K.W.; Gerke, D.S.; Herviou, L.; Ianculescu, I.; Chodankar, R.; Siegmund, K.D.; Stallcup, M.R. G9a functions as a molecular scaffold for assembly of transcriptional coactivators on a subset of glucocorticoid receptor target genes. Proc. Natl. Acad. Sci. U. S. A. 2012, 109, 19673-19678. doi:10.1073/pnas.1211803109

19. Chaturvedi, C.P.; Hosey, A.M.; Palii, C.; Perez-Iratxeta, C.; Nakatani, Y.; Ranish, J.A.; Dilworth, F.J.; Brand, M. Dual role for the methyltransferase G9a in the maintenance of beta-globin gene transcription in adult erythroid cells. Proc. Natl. Acad. Sci. U. S. A. 2009, 106, 18303-18308. doi:10.1073/pnas.0906769106

20. Lee, D.Y.; Northrop, J.P.; Kuo, M-H.; Stallcup, M.R. Histone H3 lysine 9 methyltransferase G9a is a transcriptional coactivator for nuclear receptors. J. Biol. Chem. 2006, 281, 8476-8485. doi:10.1074/jbc.M511093200

21. Schaefer, A.; Sampath, S.C.; Intrator, A.; Min, A.; Gertler, T.S.; Surmeier, D.J.; Tarakhovsky, A.; Greengard, P. Control of cognition and adaptive behavior by the GLP/G9a epigenetic suppressor complex. Neuron 2009, 64, 678-691. doi:10.1016/j.neuron.2009.11.019

22. Shankar, S.R.; Bahirvani, A.G.; Rao, V.K.; Bharathy, N.; Ow, J.R.; Taneja, R. G9a, a multipotent regulator of gene expression. Epigenetics 2013, 8, 16-22. doi: 10.4161/epi.23331

23. Scheer, S.; Zaph, C. The lysine methyltransferase G9a in immune cell differentiation and function. Front. Immunol. 2017, 8, 429. doi: 10.3389/fimmu.2017.00429

24. Tachibana, M.; Matsumura, Y.; Fukuda, M.; Kimura, H.; Shinkai, Y. G9a/GLP complexes independently mediate H3K9 and DNA methylation to silence transcription. EMBO J. 2008, 27, 2681-2690. doi:10.1038/emboj.2008.192

25. Purcell, D.J.; Jeong, K.W.; Bittencourt, D.; Gerke, D.S.; Stallcup, M.R. A distinct mechanism for coactivator versus corepressor function by histone methyltransferase G9a in transcriptional regulation. J. Biol. Chem. 2011, 286, 41963-41971. doi:10.1074/jbc.M111.298463

26. Shinkai, Y.; Tachibana, M. H3K9 methyltransferase G9a and the related molecule GLP. Genes Dev. 2011, 25, 781-788. doi: 10.1101/gad.2027411

27. Casciello, F.; Windloch, K.; Gannon, F.; Lee, J.S. Functional role of G9a histone methyltransferase in cancer. Front. Immunol. 2015, 6, 487. doi: 10.3389/fimmu.2015.00487

28. Chen, M.W.; Hua, K.T.; Kao, H.J.; Chi, C.C.; Wei, L.H.; Johansson, G.; Shiah, S.G.; Chen, P.S.; Jeng, Y.M.; Cheng, T.Y.; et al. H3K9 histone methyltransferase G9a promotes lung cancer invasion and metastasis by silencing the cell adhesion molecule Ep-CAM. Cancer Res. 2010, 70, 7830-7840. doi:10.1158/0008-5472.CAN-10-0833

29. Egger, G.; Liang, G.; Aparicio, A.; Jones, P.A. Epigenetics in human disease and prospects for epigenetic therapy. Nature 2004, 429, 457-463. doi:10.1038/nature02625

30. Hua, K.T.; Wang, M.Y.; Chen, M.W.; Wei, L.H.; Chen, C.K.; Ko, C.H.; Jeng, Y.M.; Sung, P.L.; Jan, Y.H.; Hsiao, M.; et al. The H3K9 methyltransferase G9a is a marker of aggressive ovarian cancer that promotes peritoneal metastasis. Mol. Cancer 2014, 13, 189. doi:10.1186/1476-4598-13-189

31. Wozniak, R.J.; Klimecki, W.T.; Lau, S.S.; Feinstein, Y.; Futscher, B.W. 5-Aza-2'-deoxycytidine-mediated reductions in G9A histone methyltransferase and histone $\mathrm{H} 3 \mathrm{~K} 9$ di-methylation levels are linked to tumor suppressor gene reactivation. Oncogene 2007, 26, 77-90. doi:10.1038/sj.onc.1209763

32. Zhong, X.; Chen, X.; Guan, X.; Zhang, H.; Ma, Y.; Zhang, S.; Wang, E.; Zhang, L.; Han, Y. Overexpression of G9a and MCM7 in oesophageal squamous cell carcinoma is associated with poor prognosis. Histopathology, 2015, 66, 192-200. doi:10.1111/his.12456

33. Roesler, R.; Brunetto, A.T.; Abujamra, A.L.; de Farias, C.B.; Brunetto, A.L.; Schwartsmann, G. Current and emerging molecular targets in glioma. Expert Rev. Anticancer Ther. 2010, 10, 1735-1751. doi: 10.1586/era.10.167

34. Stupp, R.; Mason, W.P.; van den Bent, M.J.; Weller, M.; Fisher, B.; Taphoorn, M.J.; Belanger, K.; Brandes, A.A.; Marosi, C.; Bogdahn, U.; et al. Radiotherapy plus concomitant and adjuvant temozolomide for glioblastoma. N. Engl. J. Med. 2005, 352, 987996. doi: 10.1056/NEJMoa043330

35. Wen, P.Y.; Kesari, S. Malignant gliomas in adults. N Engl J Med 2008, 359, 492-507. doi: 10.1056/NEJMra0708126

36. Wu, G.; Diaz, A.K.; Paugh, B.S.; Rankin, S.L.; Ju, B.; Li, Y.; Zhu, X.; Qu, C.; Chen, X.; Zhang, J., et al. The genomic landscape of diffuse intrinsic pontine glioma and pediatric non-brainstem high-grade glioma. Nat. Genet. 2014, 46, 444-450. doi: 10.1038/ng.2938

37. Venneti, S.; Felicella, M.M.; Coyne, T.; Phillips, J.J.; Gorovets, D.; Huse, J.T.; Kofler, J.; Lu, C.; Tihan, T.; Sullivan, L.M.; et al. Histone 3 lysine 9 trimethylation is differentially associated with isocitrate dehydrogenase mutations in oligodendrogliomas and high-grade astrocytomas. J. Neuropathol. Exp. Neurol. 2013, 72, 298-306. doi: 10.1097/NEN.0b013e3182898113 
38. Kunadis, E.; Lakiotaki, E.; Korkolopoulou, P.; Piperi, C. Targeting post-translational histone modifying enzymes in glioblastoma. Pharmacol. Ther. 2021, 220, 107721. doi: 10.1016/j.pharmthera.2020.107721

39. Guo, A.S.; Huang, Y.Q.; Ma, X.D.; Lin, R.S. Mechanism of G9a inhibitor BIX-01294 acting on U251 glioma cells. Mol. Med. Rep. 2016, 14, 4613-4621. doi: 10.3892/mmr.2016.5815

40. Cartron, P.F.; Blanquart, C.; Hervouet, E.; Gregoire, M.; Vallette, F.M. HDAC1-mSin3a-NCOR1, Dnmt3b-HDAC1-Egr1 and Dnmt1-PCNA-UHRF1-G9a regulate the NY-ESO1 gene expression. Mol. Oncol. 2013, 7, 452-463. doi: 10.1016/j.molonc.2012.11.004

41. Ke, X.X.; Zhang, R.; Zhong, X.; Zhang, L.; Cui, H. Deficiency of G9a inhibits cell proliferation and activates autophagy via transcriptionally regulating c-Myc expression in glioblastoma. Front. Cell Dev. Biol. 2020, 8, 593964. doi: 10.3389/fcell.2020.593964

42. Ciechomska, I.A.; Przanowski, P.; Jackl, J.; Wojtas, B.; Kaminska, B. BIX01294, an inhibitor of histone methyltransferase, induces autophagy-dependent differentiation of glioma stem-like cells. Sci. Rep. 2016, 6, 38723. doi: 10.1038/srep38723

43. Ciechomska, I.A.; Marciniak, M.P.; Jackl, J.; Kaminska, B. Pre-treatment or post-treatment of human glioma cells with BIX01294, the inhibitor of histone methyltransferase G9a, sensitizes cells to temozolomide. Front. Pharmacol. 2018, 9, 1271. doi: 10.3389/fphar.2018.01271

44. Ciechomska, I.A.; Jayaprakash, C.; Maleszewska, M.; Kaminska, B. Histone modifying enzymes and chromatin modifiers in glioma pathobiology and therapy responses. Adv. Exp. Med. Biol. 2020, 1202, 259-279. doi: 10.1007/978-3-030-30651-9_13

45. Gursoy-Yuzugullu, O.; Carman, C.; Serafim, R.B.; Myronakis, M.; Valente, V.; Price, B.D. Epigenetic therapy with inhibitors of histone methylation suppresses DNA damage signaling and increases glioma cell radiosensitivity. Oncotarget 2017, 8, 2451824532. doi: 10.18632/oncotarget

46. Ahmad, F.; Dixit, D.; Joshi, S.D.; Sen, E. G9a inhibition induced PKM2 regulates autophagic responses. Int. J. Biochem. Cell Biol. 2016, 78, 87-95. doi: 10.1016/j.biocel.2016.07.009

47. Bao, L.; Chen, Y.; Lai, H.T.; Wu, S.Y.; Wang, J.E.; Hatanpaa, K.J.; Raisanen, J.M.; Fontenot, M.; Lega, B.; Chiang, C.M.; et al. Methylation of hypoxia-inducible factor (HIF)-1 $\alpha$ by G9a/GLP inhibits HIF-1 transcriptional activity and cell migration. Nucleic Acids Res. 2018, 46, 6576-6591. doi: 10.1093/nar/gky449

48. Li, Q.; Dong, C.; Cui, J.; Wang, Y.; Hong, X. Over-expressed lncRNA HOTAIRM1 promotes tumor growth and invasion through up-regulating HOXA1 and sequestering G9a/EZH2/Dnmts away from the HOXA1 gene in glioblastoma multiforme. J. Exp. Clin. Cancer Res. 2018, 37, 265. doi: 10.1186/s13046-018-0941-x

49. Ghildiyal, R.; Sen, E. Concerted action of histone methyltransferases G9a and PRMT-1 regulates PGC-1 $\alpha$-RIG-I axis in IFN $\gamma$ treated glioma cells. Cytokine 2017, 89, 185-193. doi: 10.1016/j.cyto.2015.12.008

50. Tao, H.; Li, H.; Su, Y.; Feng, D.; Wang, X.; Zhang, C.; Ma, H.; Hu, Q. Histone methyltransferase G9a and H3K9 dimethylation inhibit the self-renewal of glioma cancer stem cells. Mol. Cell Biochem. 2014, 394, 23-30. doi: 10.1007/s11010-014-2077-4

51. Northcott, P.A.; Buchhalter, I.; Morrissy, A.S.; Hovestadt, V.; Weischenfeldt, J.; Ehrenberger, T.; Gröbner, S.; Segura-Wang, M.; Zichner, T.; Rudneva, V.A.; et al. The whole-genome landscape of medulloblastoma subtypes. Nature 2017, 547, 311-317. doi: 10.1038/nature22973

52. Taylor, M.D.; Northcott, P.A.; Korshunov, A.; Remke, M.; Cho, Y.J.; Clifford, S.C.; Eberhart, C.G.; Parsons, D.W.; Rutkowski, S.; Gajjar, A.; et al. Molecular subgroups of medulloblastoma: the current consensus. Acta Neuropathol. 2012, 123, 465-472. doi: 10.1007/s00401-011-0922-z

53. Northcott, P.A.; Korshunov, A.; Pfister, S.M.; Taylor, M.D. The clinical implications of medulloblastoma subgroups. Nat. Rev. Neurol. 2012, 8, 340-351. doi: 10.1038/nrneurol.2012.78

54. Ramaswamy, V.; Remke, M.; Bouffet, E.; Bailey, S.; Clifford, S.C.; Doz, F.; Kool, M.; Dufour, C.; Vassal, G.; Milde, T. Risk stratification of childhood medulloblastoma in the molecular era: the current consensus. Acta Neuropathol. 2016, 131, 821-831. doi: 10.1007/s00401-016-1569-6

55. Northcott, P.A.; Robinson, G.W.; Kratz, C.P.; Mabbott, D.J.; Pomeroy, S.L.; Clifford, S.C.; Rutkowski, S.; Ellison, D.W.; Malkin, D.; Taylor, M.D.; et al. Medulloblastoma. Nat. Rev. Dis. Primers 2019, 5, 11. https://doi.org/10.1038/s41572-019-0063-6

56. von Bueren, A.O.; Kortmann, R.D.; von Hoff, K.; Friedrich, C.; Mynarek, M.; Müller, K.; Goschzik, T.; Zur Mühlen, A.; Gerber, N.; Warmuth-Metz, M.; et al. Treatment of children and adolescents with metastatic medulloblastoma and prognostic relevance of clinical and biologic parameters. J. Clin. Oncol. 2016, 34, 4151-4160. doi: 10.1200/JCO.2016.67.2428

57. Rossi, A.; Caracciolo, V.; Russo, G.; Reiss, K.; Giordano, A. Medulloblastoma: from molecular pathology to therapy. Clin. Cancer Res. 2008, 14, 971-976. https://doi.org/10.1158/1078-0432.CCR-07-2072

58. Thomaz, A.; Jaeger, M.; Brunetto, A.L.; Brunetto, A.T.; Gregianin, L.; de Farias, C.B.; Ramaswamy, V.; Nör, C.; Taylor, M.D.; Roesler, R. Neurotrophin signaling in medulloblastoma. Cancers (Basel) 2020, 12, 2542. doi: 10.3390/cancers12092542

59. Dobson, T.H.W.; Hatcher, R.J.; Swaminathan, J.; Das, C.M.; Shaik, S.; Tao, R.H.; Milite, C.; Castellano, S.; Taylor, P.H.; Sbardella, G.; et al. Regulation of USP37 expression by REST-associated G9a-dependent histone methylation. Mol. Cancer Res. 2017, 15, 1073-1084. doi: 10.1158/1541-7786.MCR-16-0424

60. Zwergel, C.; Romanelli, A.; Stazi, G.; Besharat, Z.M.; Catanzaro, G.; Tafani, M.; Valente, S.; Mai, A. Application of small epigenetic modulators in pediatric medulloblastoma. Front. Pediatr. 2018, 6, 370. doi: 10.3389/fped.2018.00370

61. Miele, E.; Valente, S.; Alfano, V.; Silvano, M.; Mellini, P.; Borovika, D.; Marrocco, B.; Po, A.; Besharat, Z.M.; Catanzaro, G.; et al. The histone methyltransferase EZH2 as a druggable target in SHH medulloblastoma cancer stem cells. Oncotarget 2017, 8, 6855768570. doi: 10.18632/oncotarget.19782 
62. Cavalli, F.M.G., Remke, M., Rampasek, L., Peacock, J., Shih, D.J.H., Luu, B., Garzia, L., Torchia, J., Nör, C., Morrissy, A.S., et al. Intertumoral heterogeneity within medulloblastoma subgroups. Cancer Cell 2017, 31, 737-754.e6. doi: 10.1016/j.ccell.2017.05.005

63. Brodeur, G.M. Neuroblastoma: biological insights into a clinical enigma. Nat. Rev. Cancer 2003, 3, 203-216. doi: 10.1038/nrc1014

64. Maris, J.M.; Hogarty, M.D.; Bagatell, R.; Cohn, S.L. Neuroblastoma. Lancet 2007, 369, 2106-2120. doi: 10.1016/S01406736(07)60983-0

65. Mishra, A.; Beniwal, M.; Nandeesh, B.N.; Srinivas, D.; Somanna, S. Primary pediatric intracranial neuroblastoma: a report of two cases. J. Pediatr. Neurosci. 2018, 13, 366-370. doi: 10.4103/JPN.JPN_68_18

66. Shah, N.R.; Wilt, E.; Lobel, J.S.; Mahmud, F.; Cera, P.J. Intracranial neuroblastoma. Med. Pediatr. Oncol. 1995, 24, 257-261. doi: 10.1002/mpo.2950240408

67. Wiegel, B.; Harris, T.M.; Edwards, M.K.; Smith, R.R.; Azzarelli, B. MR of intracranial neuroblastoma with dural sinus invasion and distant metastases. AJNR Am. J. Neuroradiol. 1991, 12, 1198-1200

68. Almeida, V.R.; Vieira, I.A.; Buendia, M.; Brunetto, A.T.; Gregianin, L.J.; Brunetto, A.L.; Klamt, F.; de Farias, C.B.; Abujamra, A.L.; Lopez PLDC, et al. Combined treatments with a retinoid receptor agonist and epigenetic modulators in human neuroblastoma cells. Mol. Neurobiol. 2017, 54, 7610-7619. doi: 10.1007/s12035-016-0250-3

69. Hoebeeck, J.; Michels, E.; Pattyn, F.; Combaret, V.; Vermeulen, J.; Yigit, N.; Hoyoux, C.; Laureys, G.; De Paepe, A.; Speleman, F.; et al. Aberrant methylation of candidate tumor suppressor genes in neuroblastoma. Cancer Lett. 2009, 273, 336-346. doi: 10.1016/j.canlet.2008.08.019

70. Ke, X.X.; Zhang, D.; Zhu, S.; Xia, Q.; Xiang, Z.; Cui, H. Inhibition of H3K9 methyltransferase G9a repressed cell proliferation and induced autophagy in neuroblastoma cells. PLoS One 2014, 9, e106962. doi: 10.1371/journal.pone.0106962

71. Senyildiz, M.; Karaman, E.F.; Bas, S.S.; Pirincci, P.A.; Ozden, S. Effects of BPA on global DNA methylation and global histone 3 lysine modifications in SH-SY5Y cells: An epigenetic mechanism linking the regulation of chromatin modifiying genes. Toxicol In Vitro 2017, 44, 313-321. doi: 10.1016/j.tiv.2017.07.028

72. Bellamy, J.; Szemes, M.; Melegh, Z.; Dallosso, A.; Kollareddy, M.; Catchpoole, D.; Malik, K. Increased efficacy of histone methyltransferase G9a inhibitors against MYCN-amplified neuroblastoma. Front. Oncol. 2020, 10, 818. doi: 10.3389/fonc.2020.00818

73. Seier, J.A.; Reinhardt, J.; Saraf, K.; Ng, S.S.; Layer, J.P.; Corvino, D.; Althoff, K.; Giordano, F.A.; Schramm, A.; Fischer, M.; et al. Druggable epigenetic suppression of interferon-induced chemokine expression linked to MYCN amplification in neuroblastoma. J. Immunother. Cancer 2021, 9, e001335. doi: 10.1136/jitc-2020-001335

74. Liu, F.; Chen, X.; Allali-Hassani, A.; Quinn, A.M.; Wigle, T.J.; Wasney, G.A.; Dong, A.; Senisterra, G.; Chau, I.; Siarheyeva, A.; et al. Protein lysine methyltransferase G9a inhibitors: design, synthesis, and structure activity relationships of 2,4-diamino-7aminoalkoxy-quinazolines. J. Med. Chem. 2010, 53, 5844-5857. doi: 10.1021/jm100478y

75. Liu, F.; Barsyte-Lovejoy, D.; Allali-Hassani, A.; He, Y.; Herold, J.M.; Chen, X.; Yates, C.M.; Frye, S.V.; Brown, P.J.; Huang, J.; et al. Optimization of cellular activity of G9a inhibitors 7-aminoalkoxy-quinazolines. J. Med. Chem. 2011, 54, 6139-6150. doi: 10.1021/jm200903z

76. Milite, C.; Feoli, A.; Horton, J.R.; Rescigno, D.; Cipriano, A.; Pisapia, V.; Viviano, M.; Pepe, G.; Amendola, G.; Novellino, E.; et al. Discovery of a novel chemotype of histone lysine methyltransferase EHMT1/2 (GLP/G9a) inhibitors: rational design, synthesis, biological evaluation, and co-crystal structure. J. Med. Chem. 2019, 62, 2666-2689. doi: 10.1021/acs.jmedchem.8b02008

77. Chandar Charles, M.R.; Li, M.C.; Hsieh, H.P.; Coumar, M.S.; Mimicking H3 substrate arginine in the design of G9a lysine methyltransferase inhibitors for cancer therapy: a computational study for structure-based drug design. ACS Omega 2021, 6, 61006111. doi: 10.1021/acsomega.0c04710

78. Fujishiro, S.; Dodo, K.; Iwasa, E.; Teng, Y.; Sohtome, Y.; Hamashima, Y.; Ito, A.; Yoshida, M.; Sodeoka, M. Epidithiodiketopiperazine as a pharmacophore for protein lysine methyltransferase G9a inhibitors: reducing cytotoxicity by structural simplification. Bioorg. Med. Chem. Lett. 2013, 23, 733-736. doi: 10.1016/j.bmcl.2012.11.087

79. Chen, J.; Lin, X.; Park, K.J.; Lee, K.R.; Park, H.J. Identification of protoberberine alkaloids as novel histone methyltransferase G9a inhibitors by structure-based virtual screening. J. Comput. Aided Mol. Des. 2018, 32, 917-928. doi: 10.1007/s10822-018-0156-4

80. Feng, T.; Wang, H.; Zhang, X.; Sun, H.; You, Q. The discovery of novel histone lysine methyltransferase G9a inhibitors (part 1): molecular design based on a series of substituted 2,4-diamino-7- aminoalkoxyquinazoline by molecular-docking-guided 3D quantitative structure-activity relationship studies. Med. Chem. 2014, 10, 426-440. doi: 10.2174/15734064113096660068

81. Kondengaden, S.M.; Luo, L.F.; Huang, K.; Zhu, M.; Zang, L.; Bataba, E.; Wang, R.; Luo, C.; Wang, B.; Li K.K.; et al. Discovery of novel small molecule inhibitors of lysine methyltransferase G9a and their mechanism in leukemia cell lines. Eur. J. Med. Chem. 2016, 122, 382-393. doi: 10.1016/j.ejmech.2016.06.028

82. Chen, W.L.; Wang, Z.H.; Feng, T.T.; Li, D.D.; Wang, C.H.; Xu, X.L.; Zhang, X.J.; You, Q.D.; Guo, X.K. Discovery, design and synthesis of $6 \mathrm{H}$-anthra[1,9-cd]isoxazol-6-one scaffold as G9a inhibitor through a combination of shape-based virtual screening and structure-based molecular modification. Bioorg. Med. Chem. 2016, 24, 6102-6108. doi: 10.1016/j.bmc.2016.09.071

83. Zang, L.; Kondengaden, S.M.; Zhang, Q.; Li, X.; Sigalapalli, D.K.; Kondengadan, S.M.; Huang, K.; Li, K.K.; Li, S.; Xiao, Z.; et al. Structure based design, synthesis and activity studies of small hybrid molecules as HDAC and G9a dual inhibitors. Oncotarget 2017, 8, 63187-63207. doi: 10.18632/oncotarget.18730

84. Rabal, O.; San José-Enériz, E.; Agirre, X.; Sánchez-Arias, J.A.; Vilas-Zornoza, A.; Ugarte, A.; de Miguel, I.; Miranda, E.; Garate, L.; Fraga, M.; et al. Discovery of reversible DNA methyltransferase and lysine methyltransferase G9a inhibitors with antitumoral in vivo efficacy. J. Med. Chem. 2018, 61, 6518-6545. doi: 10.1021/acs.jmedchem.7b01926

570 571 572 573 574 575 
85. Ramya Chandar Charles, M.; Hsieh, H.P.; Selvaraj Coumar, M. Delineating the active site architecture of G9a lysine methyltransferase through substrate and inhibitor binding mode analysis: a molecular dynamics study. J. Biomol. Struct. Dyn. 2019, 37, 2581-2592. doi: 10.1080/07391102.2018.1491422

86. López-López, E.; Rabal, O.; Oyarzabal, J.; Medina-Franco, J.L. Towards the understanding of the activity of G9a inhibitors: an activity landscape and molecular modeling approach. J. Comput. Aided Mol. Des. 2020, 34, 659-669. doi: 10.1007/s10822-02000298-x

87. Charles, M.R.C.; Mahesh, A.; Lin, S.Y.; Hsieh, H.P.; Dhayalan, A.; Coumar, M.S. Identification of novel quinoline inhibitor for EHMT2/G9a through virtual screening. Biochimie 2020, 168, 220-230. doi: 10.1016/j.biochi.2019.11.006

88. Ivanov, D.P.; Coyle, B.; Walker, D.A.; Grabowska, A.M. In vitro models of medulloblastoma: Choosing the right tool for the job. J. Biotechnol. 2016, 236, 10-25. doi: 10.1016/j.jbiotec.2016.07.028

89. Cao, H.; Li, L.; Yang, D.; Zeng, L.; Yewei, X.; Yu, B.; Liao, G.; Chen, J. Recent progress in histone methyltransferase (G9a) inhibitors as anticancer agents. Eur. J. Med. Chem. 2019, 179, 537-546. doi: 10.1016/j.ejmech.2019.06.072

90. Chen, W.L.; Sun, H.P.; Li, D.D.; Wang, Z.H.; You, Q.D.; Guo, X.K. G9a - an appealing antineoplastic target. Curr. Cancer Drug Targets 2017, 17, 555-568. doi: 10.2174/1568009616666160512145303

91. Jan, S.; Dar, M.I.; Wani, R.; Sandey, J.; Mushtaq, I.; Lateef, S.; Syed, S.H. Targeting EHMT2/ G9a for cancer therapy: progress and perspective. Eur. J. Pharmacol. 2021, 893, 173827. doi: 10.1016/j.ejphar.2020.173827

92. Rahman, Z.; Bazaz, M.R.; Devabattula, G.; Khanm M.A.; Godugu, C. Targeting H3K9 methyltransferase G9a and its related molecule GLP as a potential therapeutic strategy for cancer. J. Biochem. Mol. Toxicol. 2021, 35, e22674. doi: 10.1002/jbt.22674

93. Charles, M.R.C.; Dhayalan, A.; Hsieh, H.P.; Coumar, M.S. Insights for the design of protein lysine methyltransferase G9a inhibitors. Future Med. Chem. 2019, 11, 993-1014. doi: 10.4155/fmc-2018-0396

94. Soumyanarayanan, U.; Dymock, B.W. Recently discovered EZH2 and EHMT2 (G9a) inhibitors. Future Med. Chem. 2016, 8, 1635-1654. doi: 10.4155/fmc-2016-0096

95. Nör, C.; de Farias, C.B.; Abujamra, A.L.; Schwartsmann, G.; Brunetto, A.L.; Roesler R. The histone deacetylase inhibitor sodium butyrate in combination with brain-derived neurotrophic factor reduces the viability of DAOY human medulloblastoma cells. Childs Nerv. Syst. 2011, 27, 897-901. doi: 10.1007/s00381-011-1439-4 